\title{
C3d Positive
}

National Cancer Institute

\section{Source}

National Cancer Institute. C3d Positive. NCI Thesaurus. Code C154106.

An indication that C3d expression has been detected in a sample. 